\title{
Study on Possibility of Processing TRIP Steel Sheet by Single Point Incremental Forming
}

\author{
Wang Jin \\ ${ }^{1}$ Department of Mechanical Engineering \\ Qingdao Technological University Qingdao, China, \\ ${ }^{2}$ Key Laboratory of Materials Processing and Die \& Mould \\ Technology,Huazhong University of Science and Technology \\ Wuhan, China jinwangqtech@163.com \\ Tao Long \\ Department of Mechanical Engineering \\ Qingdao Technological University \\ Qingdao, China
}

\author{
Wang Bao-ping \\ Department of Mechanical Engineering \\ Qingdao Technological University \\ Qingdao, China
}

\begin{abstract}
Two parts of TRIP590 steel were formed by single point incremental forming (SPIF), which wall thicknesses are theoretically incrassate and reduced respectively. The wall thicknesses of the parts along the depth were measured. The experimental results show that SPIF can be employed to process TRIP steel sheet, and the wall thicknesses of the two formed parts can both be divided into three segments along the depth. In addition , the incremental forming limit angle of TRIP590 steel with $0.67 \mathrm{~mm}$ in thickness was obtained to be about $66.5^{\circ}$.
\end{abstract}

Keywords- TRIP steel; incremental sheet forming; thickness; forming limit angle

\section{INTRODUCTION}

Single Point Incremental Forming (SPIF) is a new forming process that can deform sheet metal without a die. Fig. 1 shows a simple schematic diagram of SPIF. In SPIF, the sheet metal is clamped by a clamping blank and a holding blank and formed layer by layer by a moving forming tool which is controlled by a preprogrammed NC machine code[1].

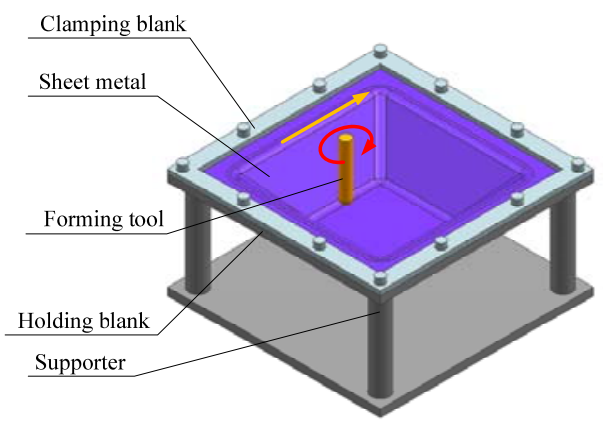

Figure.1 Schematic diagram of SPIF

This work was supported by the National Science Foundation of China under Grant No.51205217 and the State Key Laboratory of Materials Processing and Die \& Mould Technology Foundation of Huazhong University of Science and Technology under Grant No. 2011-P07 and the Project of Shandong Province Higher Educational Science and Technology Program under Grant No. J10LD13.

\author{
Jiang Hu-seng \\ Department of Mechanical Engineering \\ Qingdao Technological University \\ Qingdao, China
}

SPIF which is more suitable for manufacturing the single part and the small lot production than conventional stamping has been received more attention[2-3]. With the gradual improvement of researching into basic issues in SPIF such as forming mechanism, computing forming force and controlling forming precision, people become to pay more attention to forming different materials by SPIF, especially for some lightweight metals such as aluminium alloy, magnesium alloy and titanium alloy[4-5]. High strength steel has a wide application foreground in car making industry. But there have few research reports about processing high strength steel by SPIF, especially for TRIP steel.

The objective of this investigation was to estimate possibility of processing TRIP steel by SPIF. Two kinds of parts of a TRIP steel which wall thicknesses are theoretically incrassate and reduced respectively were formed. The wall thicknesses of the formed parts were measured, and the forming limit angle of the tested steel was obtained. The finished work will provide technical support to TRIP steel production made by SPIF.

\section{EXPERIMENTAL PROCEDURE}

The TRIP steel sheet with $0.67 \mathrm{~mm}$ in thickness of nominal composition of (mass percent, \%) C 0.18 , Si 0.55, Mn 1.4, P 0.01 , S 0.01, Cr 0.8, Ni 1.2, Mo 0.6 and Fe balance, was chosen to be tested in the present study. The square specimens, with $150 \mathrm{~mm}$ in length, were machined. Square grids with a length of $2 \mathrm{~mm}$ were etched on the surface of each specimen. A truncated cone which generatrix is a curve and a spherical crown were formed respectively (see Fig.2 and Fig.3). Major diameter with $100 \mathrm{~mm}$ and a cure with the slope ranging from $35^{\circ}-90^{\circ}$ was selected in forming the truncated cone (see Fig.2). Also, major diameter with $100 \mathrm{~mm}$ and the initial angle with $65^{\circ}$ between generatrix and the horizontal were selected in forming the spherical crown (see Fig.3). Based on the basic 
theory of SPIF, the wall thickness profile of the truncated cone decreases as depth increases. On the contrary, the wall thickness profile of the spherical crown increases as depth increases. The bulb forming tool with $10 \mathrm{~mm}$ in diameter, tool feed speed with $2000 \mathrm{~mm} / \mathrm{min}$ and each tool feed depth between two adjacent forming layers with $0.1 \mathrm{~mm}$ were used in all SPIF experiments. A machine oil was used to be the lubricant. The specimens were formed on a SPIF machine until the preprogram finished or the specimen fractured. Each specimen was marked at various points with a height vernier gauge (see Fig.4) and was cut into quarter pieces to measure the wall thickness profile.

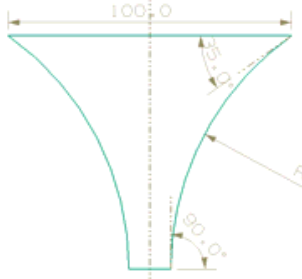

(a) Middle section

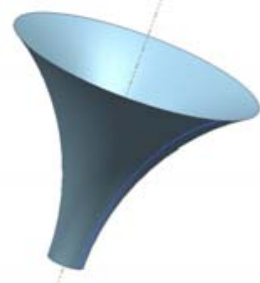

(b) 3D model
Figure.2 CAD model of the truncated cone

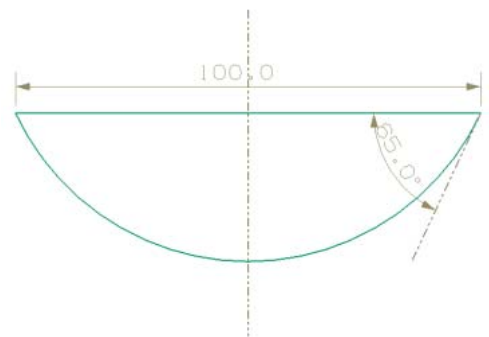

(a) Middle section

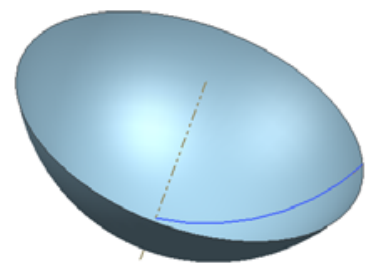

(b) 3D model

Figure.3 CAD model of the sphere crown

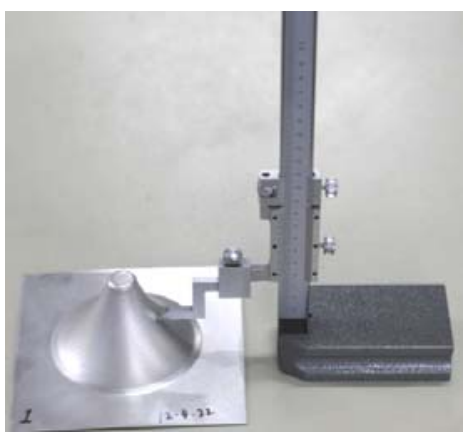

Figure.4 Marking depth on the surface of the part using a height vernier gauge

\section{EXPERIMENTAL RESULTS}

\section{A. Formed parts}

Fig.5(a) shows the formed truncated cone did not fracture until the forming depth reaches about $53 \mathrm{~mm}$. The spherical crown was formed without crack when the forming was ended (see Fig.5(b)). The results show that the TRIP steel sheet can be processed by SPIF.

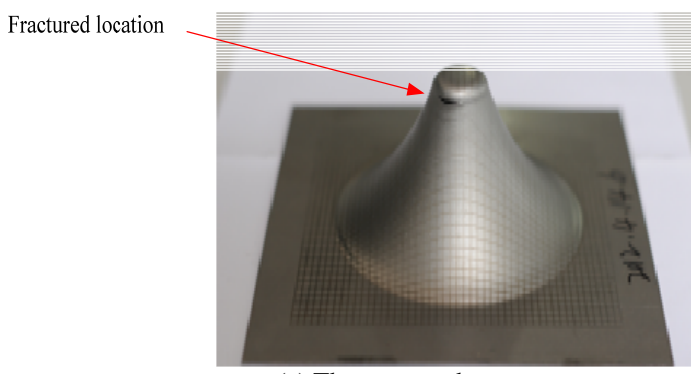

(a) The truncated cone

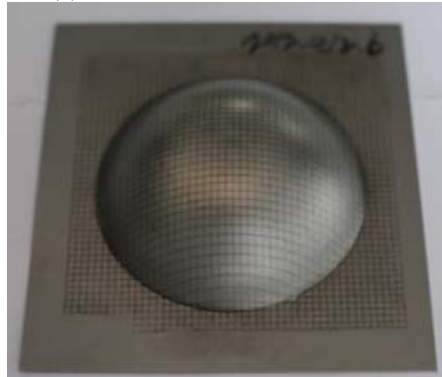

(b) The sphere crown

Figure. 5 The formed parts

\section{B. Wall thickness of parts}

The wall thickness of parts in SPIF can be expressed by the Cosin's law as follows [1]:

$$
t=t_{0} \cos (\theta)
$$

where $t$ is the wall thickness of parts in theory in SPIF, $t_{0}$ is the initial thickness of sheet, $\theta$ is the angle between the wall profile and the horizontal direction.

The wall thicknesses of the formed parts along the depth were shown in Fig.6 with the theoretical thicknesses computed by Eq.(1). The measured thicknesses of two parts can both be divided into three segments. In Fig.6(a) and Fig.6(b), the measured thickness between the point A and B is thicker than the theoretical thickness because of bending close to the clamped edge. The measured thickness of the truncated cone between the point B and C follows the Cosin's law well (see Fig.6(a)). But the actual thickness of the truncated cone after $\mathrm{C}$ decreases and deviates from the theoretical thickness as the depth increases due to the destabilization because of extra thinning of the part which fractured at the point $\mathrm{D}$ (see Fig.6(a)). Unlike the thickness distribution of the truncated cone, the actual thickness of the sphere crown is thinner than the theoretical thickness between B and C, and is in good agreement with the theoretical thickness after $C$ (see Fig.6(b)). The thinning segment in Fig.6(b) between $\mathrm{B}$ and $\mathrm{C}$ was also 
observed in other experiments when the forming angle is bigger than an angle decided by material[6].

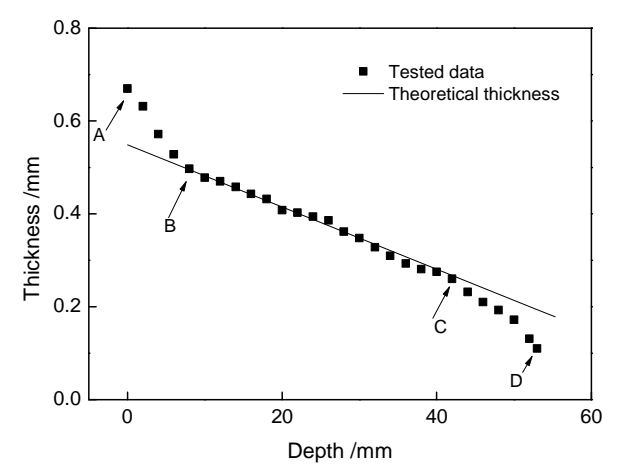

(a) The truncated cone

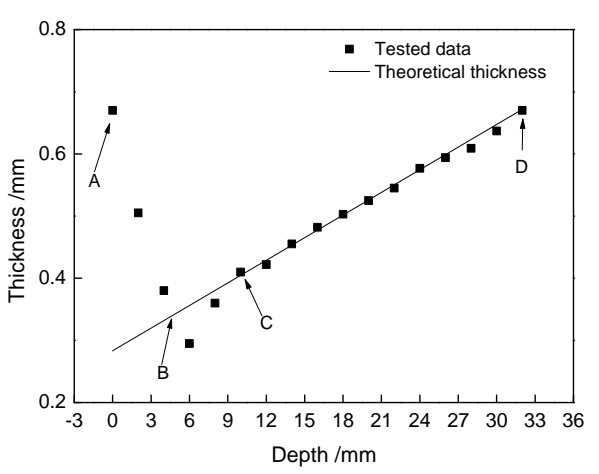

(b) The sphere crown

Figure.6 The thickness of the formed parts along the depth

\section{Forming limit angle}

According to the Eq.(1), the wall thickness of parts decreases as the forming angle increases. In SPIF, parts fracture when the forming angle is bigger than a forming limit angle which is often expressed as $\theta_{\max }$. Many experiments were performed to get the forming limit angel of different materials by processing the cone or pyramid of fixed forming angle in SPIF. The maximum angle of the fixed angle part that can be formed will be defined as the forming limit angle. Hussain $^{[7]}$ found that the fractured angle of a truncated cone which generatrix is a curve is often bigger than the forming limit angle. But the forming angle at the thickness transition point (like the point $\mathrm{C}$ in Fig.6(a)) is very close to the forming limit angle from processing fixed angle parts. Hussain[7] also deduced a formula to compute the forming angle according to the depth of a formed truncated cone which generatrix is a curve. The formula can be expressed as[7]:

$$
\theta=\cos ^{-1}\left(\frac{y_{1}-h_{D}}{R}\right)
$$

where $\theta$ is the forming angle, $y_{1}$ is the vertical distance between the opened location of the truncated cone and the center of the generatrix, $h_{D}$ is the depth at the measured point, $R$ is the radius of the generatrix.

According to the Eq.(2), the fractured wall angle of the formed truncated cone at the point D (see Fig.6(a)) was obtained to be about $73.2^{\circ}$. Followed Hussain[7], the wall angle of the formed truncated cone at the point $\mathrm{C}$ (see Fig.6(a)) is the forming limit angle of TRIP590 sheet with $0.67 \mathrm{~mm}$ in thickness in SPIF, and is about $66.5^{\circ}$.

\section{CONCLUSION}

1) Two kinds of parts of TRIP steel which wall thicknesses were incrassate and reduced respectively were formed by SPIF. The results show that the TRIP steel sheet can be processed by SPIF.

2) The wall thickness of the formed truncated cone which generatrix is a curve can be divided into three segments that are the segment thicker than the theoretical thickness, the segment equal to the theoretical thickness and the segment thinner than the theoretical thickness successively.

3) The wall thickness of the formed sphere crown can also be divided into three segments. Unlike the truncated cone, the three segments of the sphere crown are the segment thicker than the theoretical thickness, the segment thinner than the theoretical thickness and the segment equal to the theoretical thickness successively.

4) The forming limit angle of TRIP590 steel with $0.67 \mathrm{~mm}$ in thickness in SPIF was got and equal to about 66.5.

\section{REFERENCES}

[1] J. Jeswiet, F. Micari, G. Hirt, a. Bramley, J. Duflou, and J. Allwood, "Asymmetric Single Point Incremental Forming of Sheet Metal," CIRP. Ann-Manuf. Techn., vol. 54, pp. 88-114, 2005.

[2] S. B. M. Echrif, and M. Hrairi, "Research and progress in incremental sheet forming processes," Mater. Manuf. Processes., vol. 26, pp. 14041414, 2011.

[3] J. Jeswiet, "Metal forming progress since 2000," CIRP-JMST., vol. 1, pp. 2-17, 2008.

[4] G. Ambrogio, L. Filice, and F. Gagliardi, "Formability of lightweight alloys by hot incremental sheet forming," Mater. Des., vol. 34, pp. 501508, 2012.

[5] Y. Ji, and J. Park, "Formability of magnesium AZ31 sheet in the incremental forming at warm temperature," J. Mater. Process. Technol., vol. 201, pp. 354-358, 2008.

[6] L. Manco, L. Filice, and G. Ambrogio, "Analysis of the thickness distribution varying tool trajectory in single-point incremental forming," P. I. MECH. ENG. B-J. ENG., vol. 225, pp. 348-356, 2011.

[7] G. Hussain, L. Gao, and N. U. Dar, "An experimental study on some formability evaluation methods in negative incremental forming," J. Mater. Process. Technol., vol. 186, pp. 45-53, 2007. 